\title{
Monitoring of Rice Plant for Disease Detection using Machine Learning
}

\author{
Naga Swetha R, V Shravani
}

\begin{abstract}
Agriculture is an significant source of income and much of an Indian economy depends on agricultural production. Early detection of plant leaf illnesses is essential to boost crop output and profit..Agricultural specialists diagnose most illnesses through the examination of external symptoms. Farmers, however, have restricted access to professionals. This article proposes a fresh method for diagnosing and classifying rice illnesses.Four diseases were detected and categorized as bacterial blight of rice, rice blast, tungro of rice and false smut.By developing an algorithm different features such as shape, color of the Diseased leaf part were extracted. .Diseases have been Classified using SVM (Support vector machine) and classifier $k$-Nearest Neighbor (k-NN) after extracting all features. Our suggested solution also provides farmers with Diagnosis of plant disease through a scalable cooperative platform based on the Cloud. This is available via a mobile application allowing customers to send photos from various areas of the leaves that automatically diagnose real-time plant diseases.
\end{abstract}

Keywords- Artificial Intelligence, Cloud, CNN, Crop Diseases, Classifiers.

\section{INTRODUCTION}

Rice crop is one of India's major plants.Most of the land region is under rice crop cultivation and is one of the primary economic development sources.Farmers face annual yield losses and economic losses owing to pests and illnesses in rice plants. The primary causes of rice diseases are bacteria, fungi, and viruses.There are various rice diseases, four were researched in this article, namely bacterial rice blight (RBB), blast disease, tungro and false smut diseases. These illnesses have some comparable signs that cannot be understood by a common farmer eye. Farmers cannot detect signs of certain illnesses.Farmers must take help by specialists to find health of plant which is very costly and time taken to diagnose is very high.This generates the need for image processing methods to automatically detect illnesses.Generally speaking, Processing of any image follow the order of steps in identifying the illnesses: (a)Plant Image collection (b)Processing of Image to reduce size (c) Image Segmentation (d)Extraction of Feature values (e)Classifying processs. Different methods for segmentation, extraction of features and different classifiers are available for classification.

Revised Manuscript Received on February 05, 2020.

* Correspondence Author

Naga Swetha R, Asst.Prof,ECE Dept., Anurag Group of Institution, Hyderabad, India.

V Shravani, M.Tech Student,ECE Dept., Anurag Group of Institution, Hyderabad, India.

(C) The Authors. Published by Blue Eyes Intelligence Engineering and Sciences Publication (BEIESP). This is an open access article under the CC BY-NC-ND license (http://creativecommons.org/licenses/by-nc-nd/4.0/)
Advances in the fields of smart phones, cloud technology and Machine learning provide an optimal chance to create a reliable cheap method for commonly deployed crop illnesses.Mobile devices with internet connectivity have become omnipresent in fast growing countries like India. Low-cost smart phones enabled by camera and GPS are accessible extensively that could be the case used to upload geo-located pictures by people. They can communicate with more sophisticated cloud-based backend services across widely available mobile networks that can perform loads of work and capacity of high storage of data.The other technological breakthrough over the last few years is an estimation of images based on AI that has exceeded manual skills and image identification and classification can be accurate.

\section{DISEASE SYMPTOMS}

\section{- $\quad$ Bacterial Blight of rice(RBB)}

Xanthomonas Oryza pm is the creation of bacterial blight. Initial symptoms of bacterial blight may include dark brown necrotic (dead) leaf spots with yellow halos. More advanced symptoms include necrotic blotches starting at the leaf margins and advancing inward, as well as black streaking on twigs.

\section{- $\quad$ Rice Blast disease(RB)}

This is a fungal disease. Different color spots or lesions at an initial stage with dark green boundaries. More established leaf injuries are Spindle or elliptical, whitish to gray centers shown in Fig.1(b) with red to brown or necrotic border. Spots at each end are usually lengthened and pointed.

\section{Rice Tungro}

It causes discoloration of the leaf, stunted growth, decreased numbers of tiller and sterile or partially filled grains. Leafhoppers that feed on tungro-in fected crops transmit tungro disease viruses from one plant to another. The green leafhopper is the most effective vector. By feeding on it, even for a short time, leafhoppers can obtain viruses from any portion of the infected plant.It can then convey the viruses within 5-7 days to other crops instantly. The viruses do not stay in the body of the leafhopper unless it feeds on an infected plant again and acquires the viruses again.

\section{False smut}

False smut causes grain chalkiness, leading to reduced grain weight. It also reduces germination of seeds.

The disease can happen in regions of elevated relative humidity and 25-35 degree temperatures. 


\section{Monitoring of Rice Plant for Disease Detection using Machine Learning}

Gases like high levels of nitrogen content soils also promote the growth of disease. Wind can spread plant-toplant fungal sporec.

False smut is only noticeable after exertion of panicle. During the flowering phase, it can infect the plant. Plants infected with fake smut are converted into a mass of spore balls by individual rice grain.
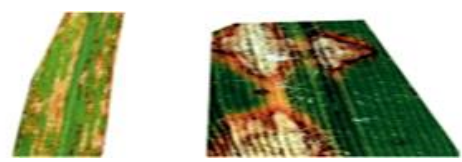

(a)

(b)

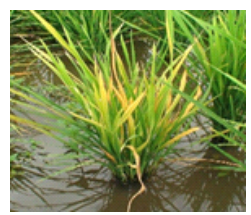

(c)

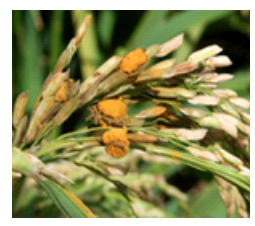

(d)

Fig.1 Rice disease signs (a) RBB, (b) RB, (c) rice tungro and (d) false smut

\section{PROPOSED METHODOLOGY}

Rice disease identification and classification methodology includes processing of image to reduce size, Thresholding method of segmentation, Finding feature values, classification of disease using different algorithms..By calculating the feature values and saving in database, some amount of pictures were taught in the training stage.

This trained images is now used to classify the input or test pictures as reference data for the classifier.Unknown image or test image is processed during the testing phase and its function values are given to the classifier as incoming variables. During the training stage, the classifier utilizes the characteristics stored in the dataset to classify them.

In detail different steps involved in plant rice diseases detection are given as below.

\section{- $\quad$ Plant image collection and processing}

In this work we have taken some images of plant in real time. We took some other internet pictures to make up for this situation that has various sizes compared to collected pictures. It collects a total of 115 pictures of diseased rice leaves. Images sizes are reduced to a standard size which enables further processing.Standardize the image to eliminate the impact of lightning while capturing a image.

\section{- Image Segmentation}

Thresholding method of segmentation is simple most effective way of dividing an image into two sets which becomes easy while processing the image.This process converts grey scale into binary images.Different methods like Histogram equalization can be performed on images.

\section{- Extraction of Features}

After the leaves of rice become exposed with illness, the leaf color changes and various sizes spots are produced on the leaves.Therefore, the color and spots geometry of the unhealthy leaves is selected to define the diseases.

\section{- Classification of Diseases}

Two classifiers were used for the disease classifying, namely SVM and k-NN . Due to easy usage and slight output time, SVM classifier is preferable. $75 \%$ of the information is used to evaluate the efficiency of the suggested method for training purposes and $25 \%$ of the information to test data .

\section{- Design of Mobile Application}

The mobile application provides the farmer with a streamlined frontend, simple and removes the variations of the storage capacity. This allows the farmer to capture plant pictures (live mode) or select current pictures for evaluation. Generally the mobile app has few options namely authentication field, new image field, load current image, disease name field, precautions of disease by experts. The mobile app uses AWS Mobile SDK for Android for communication involving database and various mobile networks.

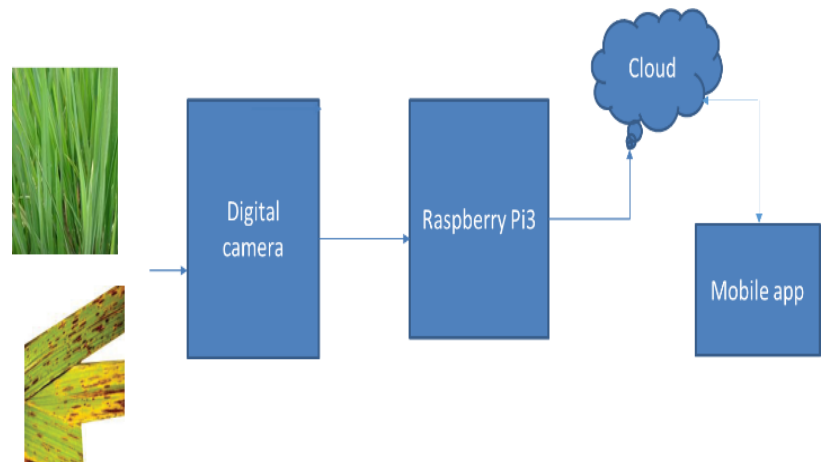

Fig 2 Overview of Plant Disease Architecture.

In the process of training the plant images which decides the disease type we use different algorithms namely convolutional neural networks . During training of images different techniques were employed. In the process of $\mathrm{CNN}$, first we perform convolution and then pooling. There will be many hidden layers which are all connected to suitable filter values of various images we considered for training.Generally in the process of training we do forward propagation of the set of images where the objective is to reduce errors each time and finally reach with least number of errors .we also perform backpropagation in which we revert back to the correct filter values which suits the particular image plant.In every step we save activation value which is the basis for image classification. 
From the plant disease architecture it is observed that we implement the whole work usng Raspberry pi3.Here in a mobile application we can enter our plant image and find out whether it is healthy or not.If the disease is unhealthy it displays the disease name and also displays the precautions to be taken immediately for diseased images.

\section{RESULTS}

The suggested technique is applied using raspberry pi3 where an output can be seen through a cloud-based mobile application whether a plant is infected or not

Table 1 Classification Accuracy of Classifiers

\begin{tabular}{|l|l|l|}
\cline { 1 - 1 } Classifiers & \multirow{2}{*}{ SVM } & K NN \\
\cline { 1 - 1 } Diseases & & \\
\hline RBB & $91.23 \%$ & $85.23 \%$ \\
\hline RB & $89.26 \%$ & $89.24 \%$ \\
\hline False Smut & $88.86 \%$ & $87.58 \%$ \\
\hline Rice Tungro & $90.23 \%$ & $89.54 \%$ \\
\hline
\end{tabular}

The used database is occupied with 120 images of various diseased rice plants, 75 percent were used to train the classifier and $25 \%$ of which were used to test. For each image, pre-processing, segmentation, extraction of features and classification steps were performed. The accuracy of classification for plant illness detection is carried out with various classifiers.

\section{CONCLUSION AND FUTURE SCOPE}

The monitoring method has been developed for bacterial blight of rice, blast disease, tungro and false smut illness of rice in this paper. To establish the classification algorithm, image processing techniques such as segmentation, extraction of features and two classifiers were used. Color along with shape characteristics were considered and taken as an input variables to the classifier which classifies the disease. .Precision with two classifiers was 91.23 percent, 89.54 percent, respectively. The proposed method is compared with other algorithms and found to be superior than others.

Extraction of color and shape features was performed in this work.In the future we can also consider various features of the leaves and can extend our work. Other rice illnesses are discussed in this job except of four mentioned diseases. Other rice diseases may be covered by future work. With few changes, the same methods can be applied to other plants.

\section{REFERENCES}

1. S. R. Maniyath et al., "Plant Disease Detection Using Machine Learning," 2018 International Conference on Design Innovations for 3Cs Compute Communicate Control (ICDI3C), Bangalore, 2018, pp. 41-45.

2. F. T. Pinki, N. Khatun and S. M. M. Islam, "Content based paddy leaf disease recognition and remedy prediction using support vector machine," 2017 20th International Conference of Computer and Information Technology (ICCIT), Dhaka, 2017, pp. 1-5.

3. B. Mishra, S. Nema, M. Lambert and S. Nema, "Recent technologies of leaf disease detection using image processing approach - A review," 2017 International Conference on Innovations in Information, Embedded and Communication Systems (ICIIECS), Coimbatore, 2017, pp. 1-5.

4. S. Phadikar and J. Sil, "Rice disease identification using pattern recognition techniques," 2008 11th International Conference on Computer and Information Technology, Khulna, 2008, pp. 420-423.

5. X. Zhou, W. Gong, W. Fu and F. Du, "Application of deep learning in object detection," 2017 IEEE/ACIS 16th International Conference on Computer and Information Science (ICIS), Wuhan, 2017, pp. 631-634.

6. S. Moedjiono, Saepudin and A. Kusdaryono, "Application Development Based On Mobile Learning Framework On Rice And Vegetable Agriculture," 2018 Third International Conference on Informatics and Computing (ICIC), Palembang, Indonesia, 2018, pp. 16.

7. T. Yu and Y. Zhu, "Research on Cloud Computing and Security," 2012 11th International Symposium on Distributed Computing and Applications to Business, Engineering \& Science, Guilin, 2012, pp. 314-316.

8. J. Liu et al., "Artificial Intelligence in the 21st Century," in IEEE Access, vol. 6, pp. 34403-34421, 2018.

9. G. Sushanth and S. Sujatha, "IOT Based Smart Agriculture System," 2018 International Conference on Wireless Communications, Signal Processing and Networking (WiSPNET), Chennai, 2018, pp. 1-4 